\title{
Biosensing-by-learning Direct Targeting Strategy for Enhanced Tumor Sensitization
}

\author{
Yifan Chen*, Muhammad Ali, Shaolong Shi, and U Kei Cheang
}

\begin{abstract}
Objective: We propose a novel iterative-optimizationinspired direct targeting strategy (DTS) for smart nanosystems, which harness swarms of externally manipulable nanoswimmers assembled by magnetic nanoparticles (MNPs) for knowledgeaided tumor sensitization and targeting. We aim to demonstrate through computational experiments that the proposed DTS can significantly enhance the accumulation of MNPs in the tumor site, which serve as a contrast agent in various medical imaging modalities, by using the shortest possible physiological routes and with minimal systemic exposure.
\end{abstract}

Methods: The epicenter of a tumor corresponds to the global maximum of an externally measurable objective function associated with an in vivo tumor-triggered biophysical gradient; the domain of the objective function is the tissue region at a high risk of malignancy; swarms of externally controllable magnetic nanoswimmers for tumor sensitization are modeled as the guess inputs. The objective function may be resulted from a passive phenomenon such as reduced blood flow or increased kurtosis of microvasculature due to tumor angiogenesis; otherwise, the objective function may involve an active phenomenon such as the fibrin formed during the coagulation cascade activated by tumortargeted "activator" nanoparticles. Subsequently, the DTS can be interpreted from the iterative optimization perspective: guess inputs (i.e., swarms of nanoswimmers) are continuously updated according to the gradient of the objective function in order to find the optimum (i.e., tumor) by moving through the domain (i.e., tissue under screening). Along this line of thought, we propose the computational model based on the gradient descent (GD) iterative method to describe the GD-inspired DTS, which takes into account the realistic in vivo propagation scenario of nanoswimmers.

Results: By means of computational experiments, we show that the GD-inspired DTS yields higher probabilities of tumor sensitization and more significant dose accumulation compared to the "brute-force" search, which corresponds to the systemic targeting scenario where drug nanoparticles attempt to target a tumor by enumerating all possible pathways in the complex vascular network.

Conclusion: The knowledge-aided DTS has potential to enhance the tumor sensitization and targeting performance remarkably by exploiting the externally measurable, tumor-triggered biophysical gradients.

Significance: We believe that this work motivates a novel biosensing-by-learning framework facilitated by externally manipulable, smart nanosystems.

Index Terms-Direct targeting strategy, biosensing-bylearning, tumor-triggered biophysical gradients, externally

Asterisk indicates corresponding author.

*Y. Chen and M. Ali are with the Faculty of Computing and Mathematical Sciences, the University of Waikato, Hamilton, New Zealand (e-mail: yifan.chen@waikato.ac.nz).

S. Shi is with Harbin Institute of Technology, Harbin, China. He is also with the Department of Computer Science and Engineering, Southern University of Science and Technology, Shenzhen, China.

U. K. Cheang is with the Department of Mechanical and Energy Engineering, Southern University of Science and Technology, Shenzhen, China. manipulable smart nanosystems, magnetic nanoswimmers, iterative optimization, natural computing, contrast-enhanced medical imaging

\section{INTRODUCTION}

\section{A. Background}

1) Contrast-enhanced Medical Imaging: Magnetic resonance imaging (MRI) is one of the standard procedures for non-invasive clinical diagnosis of cancers due to its high soft tissue contrast, spatial resolution, and penetration depth [1]. In addition, images are acquired without the use of ionizing radiation or radio tracers that would cause harmful side-effects. Contrast agents such as magnetic nanoparticles (MNPs) are commonly used in MRI to provide better delineation between healthy and diseased tissues [1]. Another promising modality for cancer diagnosis and recurrence monitoring is microwave imaging in view of its safety, mobility, and cost-effectiveness [2]. For example, a number of operational microwave breast imaging systems are already in clinical use [3]-[7] as reviewed in [8]. A major challenge faced by this approach is the potentially small dielectric contrast between tumor and its surrounding tissues, and between benign and cancerous changes [9]-[11]. To overcome these issues, MNPs have also been proposed as a contrast agent [12], [13]. However, the current systemic targeted drug delivery route can only deliver a very small fraction $(<2 \%)$ of the administered nanoparticles to the precise site [14]. The main constraints include the reliance on systemic circulation, the lack of a propelling force, and the absence of a sensory-based displacement capability [15].

2) Amplification of Tumor Homing through Externally Manipulable Nanoswimmers: Enhancing the diagnostic efficacy of contrast agents necessitates the use of a direct targeting strategy (DTS) that allows agents to reach the target tissues using the shortest physiological routes and with minimal systemic exposure. In [15], swarms of magneto-aerotactic bacteria, namely Magnetococcus marinus strain MC-1, are harnessed for delivering drug-containing nanoliposomes to the disease site to improve the therapeutic index of various nanocarriers in tumor regions. MC-1 cells, each containing a chain of magnetic iron oxide nanocrystals, tend to swim along local magnetic field lines and towards low oxygen concentrations based on a two-state aerotactic sensing system. It was shown that when $\mathrm{MC}-1$ cells were injected near the tumor and magnetically guided, up to $55 \%$ of MC- 1 cells penetrated into hypoxic regions of the tumor. Furthermore, nanoswimmers assembled by MNPs have also been proposed 
for direct targeting, which use magnetic self-assembly of $50-100 \mathrm{~nm}$ iron oxide nanoparticles [16]. Under an external magnetic field, the MNPs can magnetize and form chains that are flexible under time-varying magnetic fields via magnetohydrodynamics. A coil system was designed to actuate the nanoswimmers by applying a nearly uniform magnetic field through the Helmholtz configuration [17], [18]. One common external force can control large numbers of nanoswimmers to perform a complex task such as penetration of a tumor cell membrane for the selective release of a drug inside the cell [17], [19]. However, nanoswimmers-assisted direct targeting of contrast agents requires a priori knowledge about the location of the disease site, which is usually unavailable if the image quality is too low in the pre-contrast medical imaging. This results in a chicken-or-egg dilemma.

3) Amplification of Tumor Homing through Smart Nanosystems: Another strategy to amplify disease targeting is to design smart nanosystems that leverage the living host environment [20]-[29]. These nanosystems can be classified in two categories: environment-responsive and environment-primed [24]. The former category encompasses nanoparticles that sense and subsequently respond to their environment. Altered in vivo conditions such as redox potential, $\mathrm{pH}$, enzymatic activity, and homeostatic pathways (see Fig. 1 in [24] for a comprehensive overview of various mechanisms) induced by disease conditions can be leveraged to mobilize nanoparticle systems that are administered in these preexisting contexts. The latter category is defined by an emerging paradigm of cooperative nanosystems, such that the host environment is manipulated by an external influence to enable desired hostnanoparticle and nanoparticle-nanoparticle interactions, such as communication, recruitment, or amplification. Modifications to the host that achieve this primed environment can be accomplished by administering energy (X-rays, infrared light, heat), drugs, or nanoparticles themselves. For example, the nanosystem in [28] consists of two components. The first component is gold nanorods that populate the porous tumor vessels via systemic targeting by utilizing the conventional enhanced permeability and retention (EPR) effect and then act as photothermal antennas to specify tumor heating via remote near-infrared laser irradiation. Local tumor heating accelerates the recruitment of the second component: a targeted nanoparticle consisting of either a prototypical imaging agent (magnetofluorescent iron oxide nanoworms) or a prototypical therapeutic agent (doxorubicin-loaded liposomes). In [25], gold nanorods or engineered proteins target tumors and then locally activate the coagulation cascade to broadcast tumor location to clot-targeted nanoworms or liposomes in circulation. Smart nanosystems do not require location information of the disease site. However, they still rely on systemic circulation for homing to cancer cells without using an external guidance.

\section{B. Biosensing by Learning}

The aforementioned experimental investigations provide the basis for an externally manipulable, smart nanosystem, where non-manipulable nanoparticles can be replaced by nanoswimmers assembled by iron oxide MNPs [16], as depicted in Fig.

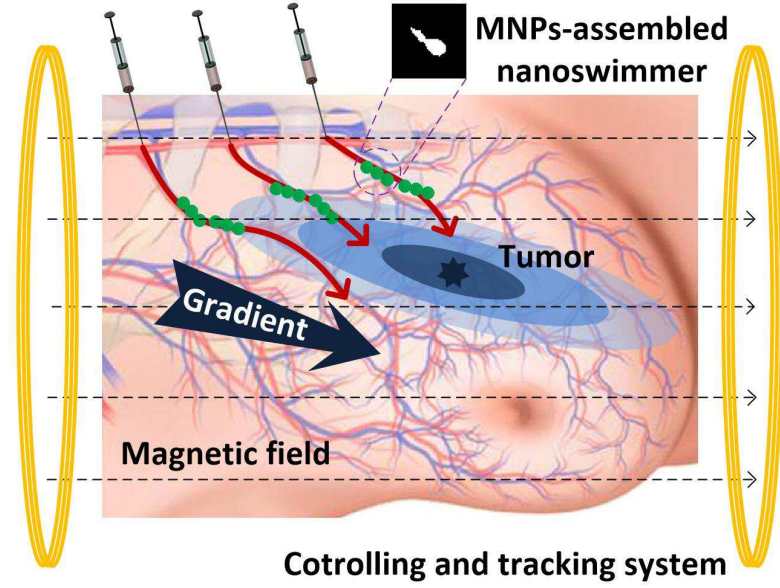

(a)

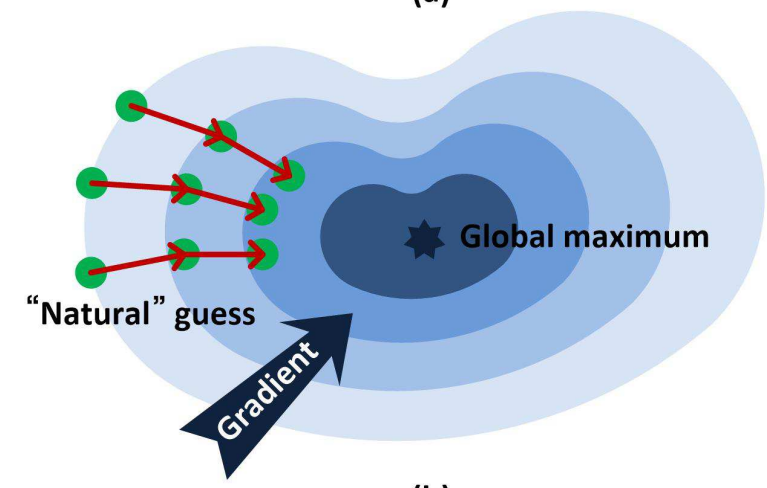

(b)

Fig. 1. Analogy between (a) DTS employed in an externally manipulable, smart nanosystem for tumor sensitization, and (b) iterative optimization process.

1(a). The magnetic response of MNPs, induced by a polarizing magnetic field, allows for reliable estimation of the locations of magnetic changes through differential medical imaging [12], [13]. For environment-responsive operations, an external controlling and tracking system probes the host environment by analyzing the measurable characteristics of nanoswimmers (e.g., trajectories, magnetic changes induced) and steers them towards the direction where a tumor is likely to be present as shown in Fig. 1(a). For environment-primed operations, gold nanorods first prime the host environment [25], [26], [28] to interact with nanoswimmers. Similarly, the external system monitors the in vivo responses by observing the properties of nanoswimmers and maneuver them correspondingly to enhance tumor sensitization. Should a specific tissue region be a tumor, MNPs will accumulate in the region on the basis of the EPR effect or receptor-ligand binding, which can be observed externally by noticing that MNPs appear to stop moving [30]-[32].

The current investigation attempts to develop a computational model for feasibility study of the proposed intelligent (i.e., knowledge-aided) DTS. Nature's blueprints have inspired exciting new fields of science such as bio-inspired computing that creates problem-solving techniques using insights from natural systems. For example, the process of natural selection inspired the development of the classical genetic algorithm to 
solve complex optimization and search problems. It is also stimulating to look the other way by exploiting computing strategies for biomedical applications [30], [31]. There is an intriguing analogy between the knowledge-aided DTS in an externally manipulable nanosystem for tumor sensitization (Fig. 1(a)) and the iterative optimization process (Fig. 1(b)). The global maximum of a unimodal, externally measurable objective function corresponding to a tumor-induced biophysical phenomenon is the tumor to be detected; the domain of the function is the tissue region at a high risk of malignancy; the guess solution is a swarm of externally manoeuvrable magnetic nanoswimmers. A guess input (i.e., nanoswimmers) locates the optimal solution (i.e., cancer) by moving through the domain (i.e., high-risk tissue) under the guidance of a specified force (i.e., steering field). The objective function may be altered by the guess made of natural materials because the guess input interacts with the domain (i.e., nanoswimmers undergo physical, chemical, and biological interactions with the host environment). This is in contrast to a traditional iterative method using a non-interacting approximate solution. An external observer can then infer the domain by monitoring the movement of the guess ("seeing-is-sensing" [31]), where the $(n+1)^{\text {th }}$ approximation is derived from the $n^{\text {th }}$ one. This strategy is within the general framework of computing-inspired bio-detection proposed in our previous work [30]. Provided with the analogy, a wide variety of iterative methods can thus be applied to the design of an optimal DTS. To elaborate on the proposed methodology, the classical gradient descent (GD) method is used to inspire the DTS, where the guess input takes steps based on the gradient of the objective function at the current point. Furthermore, the derivative of the function needs to be approximated in real-time and the movement of the guess is constrained by the physical conditions of human microvasculature.

It is worth noting that, from the computational perspective, the traditional systemic delivery of contrast agents can be regarded as a "brute-force" search where contrast agent nanoparticles attempt to detect a tumor via a medical imaging system by enumerating all possible pathways in the complex vascular network and checking whether each pathway is intercepted by a tumor. Furthermore, the original smart nanosystems in [20][29] can be regarded as a brute-force search given an expanded tumoral region due to tumor target amplification facilitated by the peritumoral biophysical conditions (for environmentresponsive nanosystems) or the initial-stage triggering modules (for environment-primed nanosystems).

\section{Organization of the Paper}

The paper is organized as follows. In Section II, we discuss the suitable in vivo biophysical gradients that can be mapped to externally measurable objective functions. In Section III, we analyze the propagation model of nanoswimmers in a discretized capillary network, followed by the discussion on the general iterative DTS framework and some representative functions showing different situations that the DTS has to face. In Section IV, we propose the GD-inspired DTS subject to the realistic physical constraints of controlling and tracking nanoswimmers in vivo. In Section $\mathrm{V}$, we provide numerical examples to demonstrate the effectiveness of the proposed framework. Finally, some concluding remarks are drawn in Section VI.

\section{Externally Measurable Objective Functions}

In the current work, tumor sensitization is performed indirectly through an external controlling and tracking system as shown in Fig. 1(a), such as an integrated device consisting of multiple pairs of electromagnetic coils to generate the rotating magnetic field to actuate the magnetic nanoswimmers [17], [18] and another coil to supply the polarizing magnetic field inducing the magnetic contrast associated with the nanoswimmers [12], [13]. Therefore, it is necessary that the in vivo biophysical gradients can be mapped to an externally measurable objective function by using nanoswimmers as a probe for analysis of the host environment.

\section{A. Environment-responsive Nanosystems}

For this type of nanosystems, passive physical properties of the host environment such as peritumoral vascular architecture [33]-[35] and blood flow velocity [34], [36]-[38] can be exploited to derive the biological gradients. Oxygen and nutrients are supplied to cancer cells via new blood vessels that have extended into the cancer tissue. Typical skeletonized images of various classes of vascular networks demonstrate that normal capillaries exhibit almost uniformly distributed grid patterns to ensure adequate oxygen transportation throughout the tissue [33]-[35]. On the other hand, tumor vessels have a profound sort of tortuosity with many smaller bends on each larger bend [33]- 35]. In terms of blood flow velocity, its value in tumor tissues is significantly lower than that in healthy tissues due to the hypovascular structure of the malignant lesion [36], [38]. This phenomenon has been observed for cancer cells in the visceral pleura [37], malignant gliomas [34], and pancreatic tumors [38]. In summary, the externally measurable objective functions corresponding to the aforementioned two biophysical conditions can be derived from the variations in the tortuosity of nanoswimmer trajectory and the resultant nanoswimmer velocity, respectively, with respect to the values for normal tissues. Both of them would increase as the distance between the nanoswimmer and the tumor decreases.

\section{B. Environment-primed Nanosystems}

For this type of systems, specific "activator" nanomaterials can be used to detect a diseased site and act as tumor-specific triggers to induce biophysical gradients. For example, gold nanorods can be modified to circulate for long periods of time in the blood stream and be passively accumulated in tumors via systemic circulation [25], [26], [28]. They are used to heat tumor tissues by amplifying the absorption of near-infrared energy that is mostly transparent to living tissues [25], [26], [28]. The associated photothermal heating is highly localized around the tumor site. Consequently, the gradient of blood flow velocity is amplified due to the differential response of normal and tumor microcirculation to hyperthermia, where blood flow 
in normal tissue increases much faster with temperature and stasis occurs at higher levels of hyperthermia compared to tumors owning to the rapid growth of tumor cell population relative to deteriorating vascular beds [39], [40]. Furthermore, local heating disrupts tumor vessels and initiates extravascular coagulation. Hence, the fibrin forms the coagulation gradient centered at the tumor caused by temperature increase. The magnetic nanoswimmers employ the peptide coatings that recognize fibrin directly for clot targeting [25], [26], [28]. Direct binding in regions of coagulation will reduce the concentration of mobile nanoswimmers under tracking. In summary, the externally measurable objective functions associated with the aforementioned two phenomena can be derived from the variations in the resultant nanoswimmer velocity and the magnetic contrast induced by nanoswimmers, respectively, with respect to the values for normal tissues. Both of them would increase as the distance between the nanoswimmer and the tumor decreases. In addition, local hyperthermia results in a temperature gradient from the tissue malignancy to its peripheral region, which may be directly measured from the infrared thermographic imaging if the tumor is close to the skin [25], [26], [28]. In this case, the global gradient towards the tumor epicenter can be readily obtained.

\section{ITERATIVE DTS}

\section{A. Invasion-percolation-based Multilayer Vascular Network Model}

Tumor vasculature is more chaotic in appearance than normal vasculature, which can be measured using fractal geometries [35]. For example, tumor vessels yield fractal dimensions of $1.89 \pm 0.04$, whereas normal arteries and veins yield dimensions of $1.70 \pm 0.03$, and normal capillaries produce essentially two-dimensional patterns [33]-[35]. It was also observed that the microvascular density in the peritumoral region increases due to the supply of growth factors from the tumor and reduces in the tumor center due to a combination of severely reduced blood flow and solid stress exerted by the tumor [41].

Consequently, it is assumed that normal tissues are regularly vascularized, which results in a homogeneous lattice comprised of straight, rigid cylindrical capillaries that join adjacent nodes [34], [42]. On the other hand, the observed fractal dimensions of tumor vasculature can be described by the invasion percolation process [33]-[35], which is implemented by first assigning uniformly distributed random values of strengths to each point on the underlying square lattice representing potential paths of vascular growth. Starting at an arbitrary site the network occupies the lattice point adjacent to the current site that has the lowest strength. Growth is iterated until the desired lattice occupancy is reached. Blood vessels are assumed to connect all adjacent occupied lattice points. Finally, additional edges are added to "pathological" nodes to ensure nonzero blood flow throughout the entire percolation cluster. The simulated networks may be matched with real tumor vasculature by selecting appropriate occupancy levels. Following [34], the fractal dimensions are around 1.6, 1.8, 1.9, and 2.0 for $40,60,80$, and $100 \%$ occupancy on the backbone, respectively.

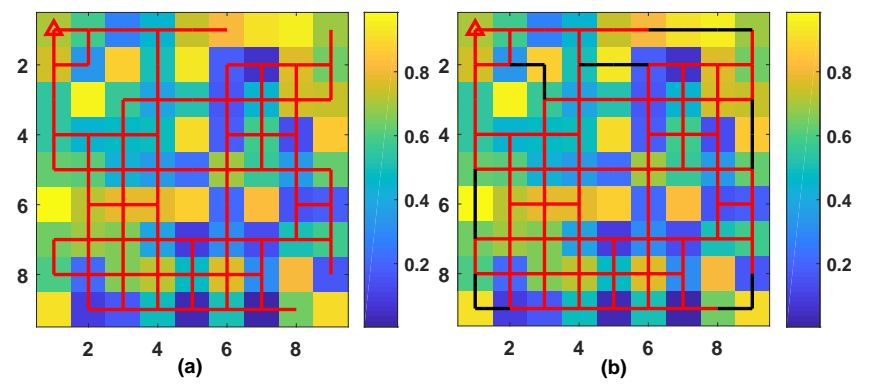

Fig. 2. (a) An invasion percolation network after 100 growth steps, and (b) adding vessels to ensure nonzero blood flow throughout the network.

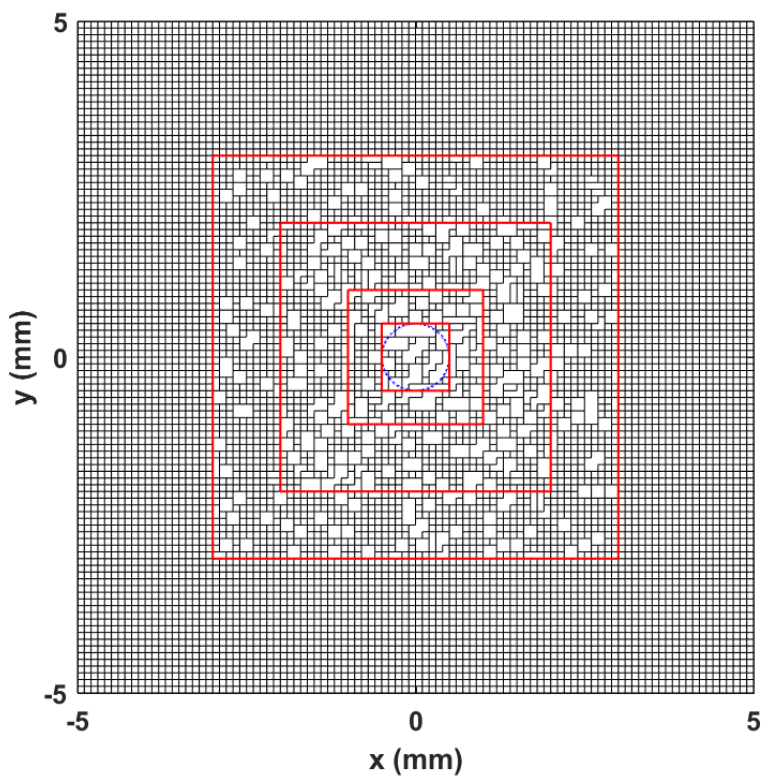

Fig. 3. Simulated multi-layer vascular network. The level of occupancy on the lattice reduces from $100 \%$ to $40 \%$ gradually as the distance to the tumor center decreases. The boundaries of the layers are denoted by the red solid lines. The tumor center is denoted by the blue dotted circle.

Moreover, malignant tumors often possess fuzzy and blurred boundaries [43], [44]. As such, the fractal dimensions across the boundary of a tumor can be characterized by a smooth transition from inside a tumor to the outside. To quantify the diffusive nature of a tissue anomaly, a discretized multilayer model can be applied to approximate the gradual, continuous change in the fractal dimension across the periphery of a lesion. Fig. 2(a) depicts an invasion percolation network after 100 growth steps, and Fig. 2(b) shows additional vessels to ensure nonzero blood flow throughout the network assuming that the blood inflow and outflow are in the top left and bottom right. Fig. 3 illustrates a simulated multilayer vascular network, where the intercapillary distance is set to be $100 \mu \mathrm{m}$, and the level of occupancy on the lattice reduces from $100 \%$ to $40 \%$ gradually as the distance to the tumor center decreases. 


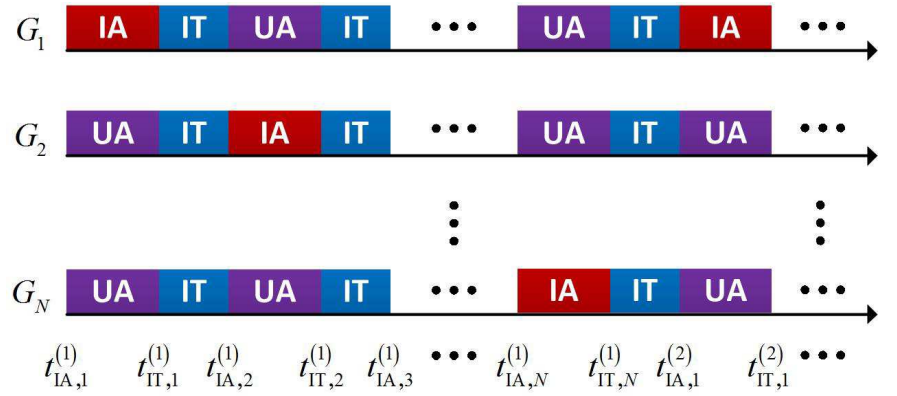

(a)

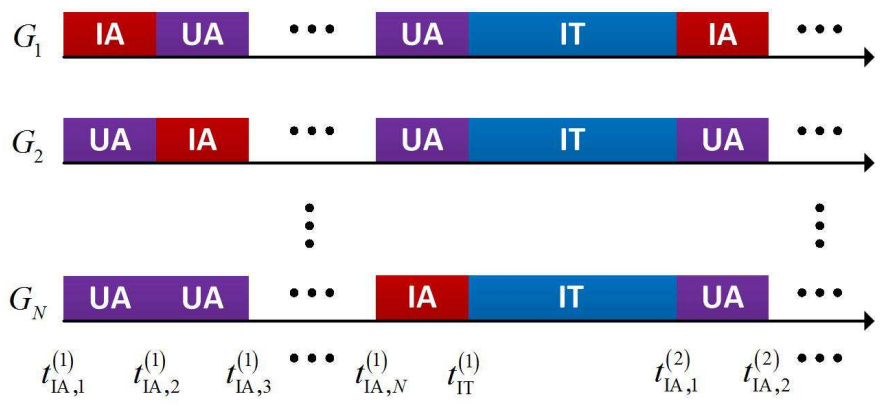

(b)

Fig. 4. Time sequence of system operational modes in one cycle for multiple guess inputs. Each guess has three operational modes: intended actuating (IA), imaging and tracking (IT), and unintended actuating (UA). Two time-division multiplexing (TDM) protocols are considered. (a) TDM1: each guess takes turn to operate in the IA and IT modes; and (b) TDM2: each guess takes turn to operate in the IA mode, followed by a common IT mode.

\section{B. Problem Formulation}

Let $f$ represent an externally measurable objective function and be defined on the domain $\mathbb{D}$, which denotes the highrisk tissue region under surveillance. The landscape of $f$ is distorted by a "natural" guess $G$ representing a swarm of magnetic nanoswimmers as follows

$$
\begin{aligned}
f(\vec{x} ; G) & =f_{\mathrm{A}}(\vec{x} ; G)+f_{\mathrm{C}}(\vec{x} ; G) \\
& =f_{\mathrm{T}}(\vec{x})+f_{\mathrm{D}}(\vec{x} ; G)+f_{\mathrm{C}}(\vec{x} ; G), \quad \vec{x} \in \mathbb{D},
\end{aligned}
$$

where $f_{\mathrm{A}}(\vec{x} ; G)$ is the apparent objective function measured at location $\vec{x}$ through guess $G, f_{\mathrm{T}}(\vec{x})$ is the true objective function at $\vec{x}$ independent of the presence or absence of $G, f_{\mathrm{D}}(\vec{x} ; G)$ is the disturbance resulted from the interaction between $G$ and the domain $\mathbb{D}$, and $f_{\mathrm{C}}(\vec{x} ; G)$ is the correction factor accounting for the disturbance caused by $G$. For a meaningful optimization process, it is assumed that regardless of any variation caused by the guess to the function, the location of the global maximum denoting the tumor, $\vec{x}^{\star}$, remains unchanged.

The true objective $f_{\mathrm{T}}(\vec{x})$, dependent on the underlying tumor-triggered biophysical phenomena, may take the form of variation in path tortuosity, velocity, or magnetic contrast of nanoswimmers as discussed earlier. Subsequently, for the measure of tortuosity, an alteration $f_{\mathrm{D}}(\vec{x} ; G)$ would incur if the nanoswimmers are engineered to modify the vasculature of tumors (e.g., anti-angiogenic agents to shut down tumor vessels or pro-angiogenic agents to normalize tumor vessels) [24].
For the measure of velocity, $f_{\mathrm{D}}(\vec{x} ; G)$ is given by the relative velocity of nanoswimmers with respect to the blood stream. In the case of magnetic change, $f_{\mathrm{D}}(\vec{x} ; G)$ is proportional to the reduction in the concentration of nanoswimmers due to various loss mechanisms such as degradation (nanoswimmers degenerate in the blood), branching (nanoswimmers move into an unintended vascular branch), and diffusion (random motions of nanoswimmers driven by the concentration gradient) [45]. Finally, the correction factor $f_{\mathrm{C}}(\vec{x} ; G)$ attempts to counteract $f_{\mathrm{D}}(\vec{x} ; G)$ to minimize its influence on the true landscape, i.e., $f_{\mathrm{C}}(\vec{x} ; G)=-f_{\mathrm{D}}(\vec{x} ; G)+\chi(\vec{x} ; G)$ with $\chi(\vec{x} ; G)$ being the random compensation error. Therefore, Eq. (1) can be rewritten as

$$
f(\vec{x} ; G)=f_{\mathrm{T}}(\vec{x})+\chi(\vec{x} ; G), \quad \vec{x} \in \mathbb{D} .
$$

In the computational framework of DTS, multiple guess inputs $G_{1}, G_{2}, \cdots, G_{N}$ are first deployed in multiple prespecified sites $\mathbb{R}_{1}, \mathbb{R}_{2}, \cdots, \mathbb{R}_{N} \subseteq \mathbb{D}$, where $\mathbb{R}_{n}$ ( $n=$ $1,2, \cdots, N)$ denote the injection sites of nanoswimmers as depicted in Fig. 1. The guesses begin searching for the optimal solution following some iterative algorithms. The DTS includes the following key steps.

1) Initialization. The guess inputs are deployed in $\mathbb{R}_{1}, \mathbb{R}_{2}, \cdots, \mathbb{R}_{N}$ at the same starting times $t_{\mathrm{IA}, 1}^{(1)}$ as shown in Fig. 4(a)-(b) with initial locations $\vec{x}_{1}\left(t_{\mathrm{IA}, 1}^{(1)}\right), \vec{x}_{2}\left(t_{\mathrm{IA}, 1}^{(1)}\right), \cdots, \vec{x}_{N}\left(t_{\mathrm{IA}, 1}^{(1)}\right), \quad$ respectively. Suppose that the external system operates in the simple time-multiplexed manner. Without loss of generality, consider the first guess input $G_{1}$, which operates on the following three modes: Intended Actuating (IA), Imaging and Tracking (IT), and Unintended Actuating (UA). Two time-division multiplexing (TDM) protocols are considered. For TDM1 each guess takes turn to operate in the IA and IT modes, whereas for TDM2 each guess takes turn to operate in the IA mode, followed by a common IT mode as illustrated in Fig. 4(a) and 4(b), respectively.

2) IA. For TDM1, from $t_{\mathrm{IA}, 1}^{(1)}$ to $t_{\mathrm{IT}, 1}^{(1)}, G_{1}$ operates in the IA mode and its trajectory is determined by the angle deviation relative to a principal axis denoting an intended steering vector upon $G_{1}$ at $\vec{x}_{1}\left(t_{\mathrm{IA}, 1}^{(1)}\right), \phi\left(t_{\mathrm{IA}, 1}^{(1)}\right)$, which indicates a uniform magnetic field in the surveillance domain [17], [18] and is dependent on the iterative method described in Section IV. The next location of $G_{1}$ at time instant $t_{\mathrm{IT}, 1}^{(1)}$ is then updated according to:

$$
\begin{aligned}
& \vec{x}_{1}\left(t_{\mathrm{IT}, 1}^{(1)}\right) \\
& =\vec{x}_{1}\left(t_{\mathrm{IA}, 1}^{(1)}\right)+d_{1}\left(t_{\mathrm{IA}, 1}^{(1)}\right) \vec{u}_{\angle\left[\phi\left(t_{\mathrm{IA}, 1}^{(1)}\right)+\Delta \phi\left(t_{\mathrm{IA}, 1}^{(1)}\right)\right]} \\
& \quad+\vec{q}_{1}\left(t_{\mathrm{IA}, 1}^{(1)}\right) .
\end{aligned}
$$

The term $\vec{u}_{\angle \phi}$ denotes a unit vector with angle $\phi$ and $\Delta \phi\left(t_{\mathrm{IA}, 1}^{(1)}\right)$ is a random variable summarizing all steering imperfections, which is assumed to be normally dis- 


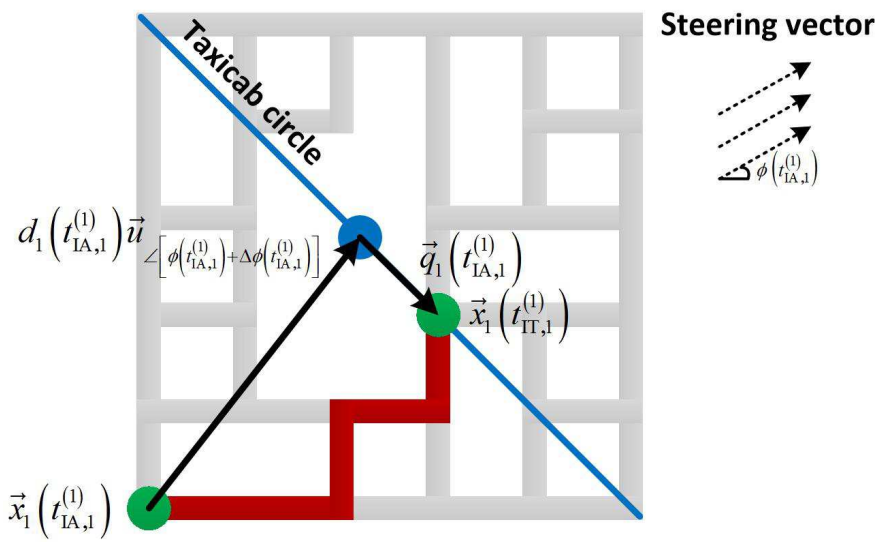

Fig. 5. Updating of a guess input in the taxicab vascular network subject to a specified steering vector.

tributed with variance $\sigma_{\Delta \phi}^{2}$ and zero mean for simplicity. The displacement length $d_{1}\left(t_{\mathrm{IA}, 1}^{(1)}\right)$ satisfies

$$
\begin{aligned}
& \left\|d_{1}\left(t_{\mathrm{IA}, 1}^{(1)}\right) \vec{u}_{\angle\left[\phi\left(t_{\mathrm{IA}, 1}^{(1)}\right)+\Delta \phi\left(t_{\mathrm{IA}, 1}^{(1)}\right)\right]}\right\|_{1} \\
& =\left|d_{1}\left(t_{\mathrm{IA}, 1}^{(1)}\right) \cos \left[\phi\left(t_{\mathrm{IA}, 1}^{(1)}\right)+\Delta \phi\left(t_{\mathrm{IA}, 1}^{(1)}\right)\right]\right| \\
& \quad+\left|d_{1}\left(t_{\mathrm{IA}, 1}^{(1)}\right) \sin \left[\phi\left(t_{\mathrm{IA}, 1}^{(1)}\right)+\Delta \phi\left(t_{\mathrm{IA}, 1}^{(1)}\right)\right]\right| \\
& =v_{1}\left(t_{\mathrm{IA}, 1}^{(1)}\right)\left(t_{\mathrm{IT}, 1}^{(1)}-t_{\mathrm{IA}, 1}^{(1)}\right),
\end{aligned}
$$

where $\|\cdot\|_{1}$ denotes the $\ell_{1}$ norm and $v_{1}\left(t_{\mathrm{IA}, 1}^{(1)}\right)$ is the velocity of $G_{1}$ at $t_{\mathrm{IA}, 1}^{(1)}$ given the taxicab geometry of the vascular network. Finally, $\vec{q}_{1}\left(t_{\mathrm{IA}, 1}^{(1)}\right)$ is the position "quantization" error due to the discrete lattice pattern of the vasculature as illustrated in Fig. 5, which is the displacement vector from the point $\vec{x}_{1}\left(t_{\mathrm{IA}, 1}^{(1)}\right)+$ $d_{1}\left(t_{\mathrm{IA}, 1}^{(1)}\right) \vec{u}_{\angle\left[\phi\left(t_{\mathrm{IA}, 1}^{(1)}\right)+\Delta \phi\left(t_{\mathrm{IA}, 1}^{(1)}\right)\right]}$ on the continuous taxicab circle of radius $v_{1}\left(t_{\mathrm{IA}, 1}^{(1)}\right)\left(t_{\mathrm{IT}, 1}^{(1)}-t_{\mathrm{IA}, 1}^{(1)}\right)$, to its closest point in the discrete vascular network having the same taxicab distance to $\vec{x}_{1}\left(t_{\mathrm{IA}, 1}^{(1)}\right)$.

For TDM2, the same process as mentioned above applies except that $t_{\mathrm{IT}, 1}^{(1)}$ is replaced by $t_{\mathrm{IA}, 2}^{(1)}$ as shown in Fig. 4(b).

3) IT. For TDM1, from $t_{\mathrm{IT}, 1}^{(1)}$ to $t_{\mathrm{IA}, 2}^{(1)}, G_{1}$ operates in the IT mode. In the absence of a steering field, $G_{1}$ follows a random walk in the lattice (i.e., at each intersection it has the same probability to either move up or to the right) and swims towards various locations in equal observation time intervals along a zigzag pathway, $\vec{x}_{1}\left(t_{\mathrm{IT}, 1}^{(1)}\right)$, $\vec{x}_{1}\left(t_{\mathrm{IT}, 1}^{(1)}+\Delta t\right), \cdots, \vec{x}_{1}\left(t_{\mathrm{IT}, 1}^{(1)}+K \Delta t\right), \vec{x}_{1}\left(t_{\mathrm{IA}, 2}^{(1)}\right)$ as shown in Fig. 6, where $\Delta t=\left(t_{\mathrm{IA}, 2}^{(1)}-t_{\mathrm{IT}, 1}^{(1)}\right) /(K+1)$. Various imaging modalities such as MRI [1] and microwave imaging [12] can be used to detect the magnetic contrast induced by multiple magnetic nanoswimmers simultaneously, which allows for tracking of all the nanoswimmers. In contrast to mathematical computing where the location of a guess input is known exactly, the guess location in the current "natural" computing needs to be estimated. The positioning error is summarized in the random variable $\Delta \vec{x}_{1}$ as also shown in Fig. 6, whose horizontal and vertical components are assumed to be independently and identically distributed Gaussian random variables with equal variance $\sigma_{\Delta x}^{2}$ and zero mean for simplicity. In that case, $\left|\Delta \vec{x}_{1}\right|$ is Rayleigh-distributed. The objective function is then evaluated at each location. For example, if the nanoswimmer velocity is considered, the values are obtained as

$$
\begin{aligned}
& f\left(\vec{x}_{1}\left(t_{\mathrm{IT}, 1}^{(1)}+k \Delta t\right)+\Delta \vec{x}_{1}\left(t_{\mathrm{IT}, 1}^{(1)}+k \Delta t\right)\right) \\
& \approx \frac{1}{\Delta t} \| \vec{x}_{1}\left(t_{\mathrm{IT}, 1}^{(1)}+(k+1) \Delta t\right) \\
& +\Delta \vec{x}_{1}\left(t_{\mathrm{IT}, 1}^{(1)}+(k+1) \Delta t\right) \\
& -\vec{x}_{1}\left(t_{\mathrm{IT}, 1}^{(1)}+k \Delta t\right) \\
& -\Delta \vec{x}_{1}\left(t_{\mathrm{IT}, 1}^{(1)}+k \Delta t\right) \|_{1}, k=0,1, \cdots, K \text {. }
\end{aligned}
$$

Subsequently, the gradient for guess $G_{1}$ at $t_{\mathrm{IT}, 1}^{(1)}$ is estimated as follows

$$
\begin{aligned}
& \nabla f\left(\vec{x}_{1}\left(t_{\mathrm{IT}, 1}^{(1)}\right)\right) \\
& \approx \max _{k_{1}, k_{2}} \\
& \left\{\left[f\left(\vec{x}_{1}\left(t_{\mathrm{IT}, 1}^{(1)}+k_{1} \Delta t\right)+\Delta \vec{x}_{1}\left(t_{\mathrm{IT}, 1}^{(1)}+k_{1} \Delta t\right)\right)\right.\right. \\
& \left.\quad-f\left(\vec{x}_{1}\left(t_{\mathrm{IT}, 1}^{(1)}+k_{2} \Delta t\right)+\Delta \vec{x}_{1}\left(t_{\mathrm{IT}, 1}^{(1)}+k_{2} \Delta t\right)\right)\right] / \\
& \| \vec{x}_{1}\left(t_{\mathrm{IT}, 1}^{(1)}+k_{1} \Delta t\right)+\Delta \vec{x}_{1}\left(t_{\mathrm{IT}, 1}^{(1)}+k_{1} \Delta t\right) \\
& \left.-\vec{x}_{1}\left(t_{\mathrm{IT}, 1}^{(1)}+k_{2} \Delta t\right)-\Delta \vec{x}_{1}\left(t_{\mathrm{IT}, 1}^{(1)}+k_{2} \Delta t\right) \|_{2}\right\}, \\
& k_{1}>k_{2} \text { and } k_{1}, k_{2} \in\{0,1, \cdots, K\},
\end{aligned}
$$

where $\|\cdot\|_{2}$ is the $\ell_{2}$ norm. The overall gradient after $N$ IT processes is estimated by taking into account all the $N$ gradients obtained at $t_{\mathrm{IT}, 1}^{(1)}, t_{\mathrm{IT}, 2}^{(1)}, \cdots, t_{\mathrm{IT}, N}^{(1)}$, respectively. A new steering vector for $G_{1}$ is then computed by following a specified algorithm as discussed in Section IV, which is used to guide the movement of $G_{1}$ during the next IA operation at $t_{\mathrm{IA}, 1}^{(2)}$ as shown in Fig. 4(a). As the nanoswimmer is in the form of nanochains or bundlelike aggregates assembled by MNPs [16], it has a finite lifespan due to the dissembling and diffusion of MNPs during propagation. In the case that $G_{1}$ is fully consumed in $\mathbb{D}$, a new guess input is deployed at $\mathbb{R}$.

For TDM2, $G_{1}$ operates in the UA mode (as explained below) from $t_{\mathrm{IA}, 2}^{(1)}$ to $t_{\mathrm{IA}, 3}^{(1)}$ when $G_{2}$ is in the IA mode.

4) UA. For TDM1, from $t_{\mathrm{IA}, 2}^{(1)}$ to $t_{\mathrm{IT}, 2}^{(1)}, G_{1}$ operates in the UA mode. This is similar to the IA operation except that the steering field is meant for the second guess $G_{2}$. 


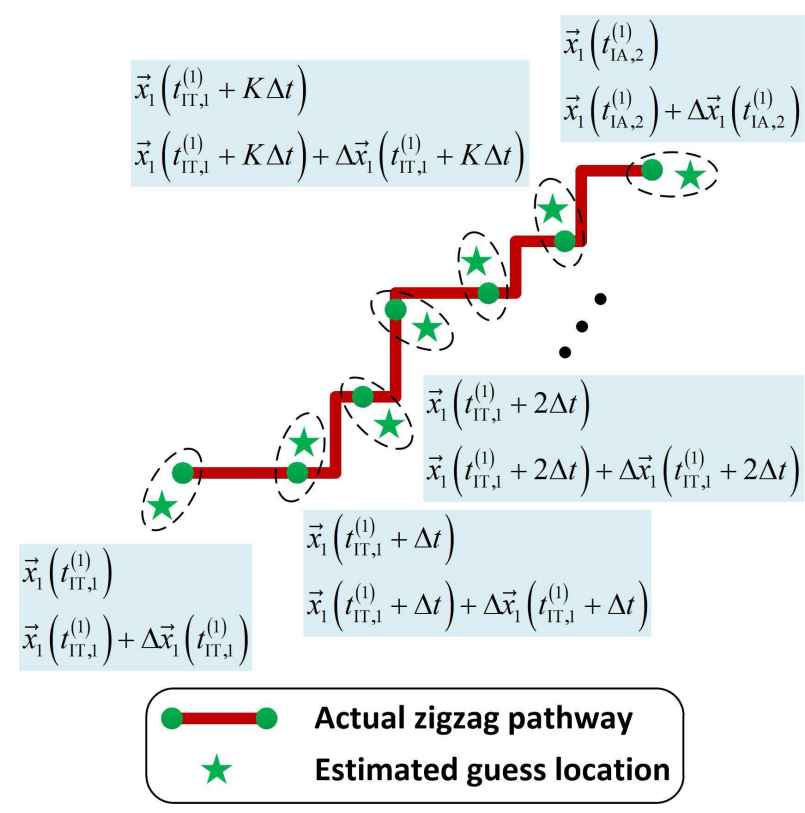

Fig. 6. Pictorial illustration of the IT process: the guess follows a random walk in the lattice and moves towards various locations along a zigzag pathway, $\vec{x}_{1}\left(t_{\mathrm{IT}, 1}\right), \vec{x}_{1}\left(t_{\mathrm{IT}, 1}+\Delta t\right), \cdots, \vec{x}_{1}\left(t_{\mathrm{IT}, 1}+K \Delta t\right), \vec{x}_{1}\left(t_{\mathrm{IA}, 2}\right)$ with $\Delta t$ being the observation time interval.

This is due to the limitation of the current coil system in generating the steering field, which exerts a global uniform torque on all the nanoswimmers simultaneously instead of localized torques on individual nanoswimmers. The same DTS steps (i.e., IA $\rightarrow$ IT $\rightarrow$ UA) are applied to all the guesses in sequence and the iteration continues unless certain stopping criteria are met.

For TDM2, from $t_{\mathrm{IA}, 3}^{(1)}$ to $t_{\mathrm{IA}, 4}^{(1)}, G_{1}$ again operates in the UA mode when $G_{3}$ is now in the IA mode. The next IT operation only occurs after all the guess inputs complete their individual IA operations. This will be followed by a new round of IA $\rightarrow$ UA $\rightarrow$ IT operations starting at $t_{\mathrm{IA}, 1}^{(2)}$ as shown in Fig. 4(b).

The mapping from an iterative optimization process in mathematical computing to the aforementioned tumor sensitization process in natural computing is illustrated in Fig. 7, which encompasses the following procedures.

1) General Mapping. Formulate the nanoswimmers-assisted tumor sensitization in the perspective of natural computing as a stylized representation of the iterative optimization problem in mathematical computing.

2) Specific Mapping. Consider a specific iterative optimization algorithm $\mathcal{A}$, which is the GD in the current work, and map $\mathcal{A}$ onto the corresponding DTS $\mathcal{S}$. For example, the key operations in a standard GD include taking the step, finding the gradient, and evaluating the objective function. The first operation corresponds to the IA mode in the DTS and the last two operations are associated with the IT mode in the DTS.

3) Reality Check. Identify the key physical constraints associated with $\mathcal{S}$ when applied in a realistic in vivo environment, compared to the original algorithm $\mathcal{A}$ when

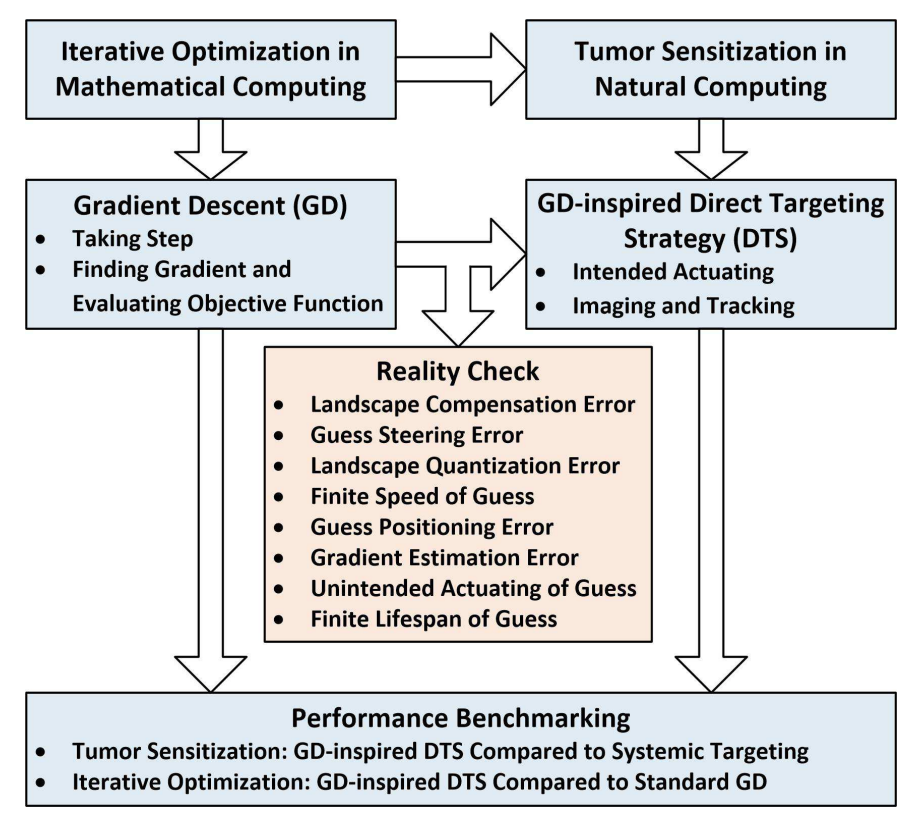

Fig. 7. Mapping from iterative optimization in mathematical computing to tumor sensitization in natural computing.

applied in an idealistic mathematical setting. For example, the imperfections in DTS include the landscape mismatch $\chi(\vec{x} ; G)$ in Eq. (2), the steering imperfection $\Delta \phi\left(t_{\mathrm{IA}, 1}^{(1)}\right)$ and the landscape quantization noise $\vec{q}_{1}\left(t_{\mathrm{IA}, 1}^{(1)}\right)$ in Eq. (3), the finite velocity of guess $v_{1}\left(t_{\mathrm{IA}, 1}^{(1)}\right)$ in Eq. (4), the positioning error $\Delta \vec{x}_{1}\left(t_{\mathrm{IT}, 1}^{(1)}+(k+1) \Delta t\right)$ and the gradient estimation inaccuracy in Eq. (5), the interference in guess update caused by UA, and the finite lifespan of guess inputs.

4) Performance Benchmarking. From the tumor sensitization perspective, we can evaluate the performance of the DTS $\mathcal{S}$ by comparing $\mathcal{S}$ to the "brute-force" systemic targeting without implementing any knowledge-aided targeting strategy. From the iterative optimization perspective, we can compare $\mathcal{S}$ to the standard algorithm $\mathcal{A}$. In this case, $\mathcal{S}$ is regarded as a degenerate form of $\mathcal{A}$.

\section{Representative Objective Functions}

As the research is in its early stage, there is no widelyaccepted, quantitative model on any of the aforementioned biophysical gradients in the existing literature other than some qualitative observations made from experimental data. As an initial investigation, three representative objective functions are considered to evaluate the performance of the externally manipulable, smart nanosystems for enhanced tumor sensitization as shown in Fig. 8. The maximum value is normalized to 1 and the minimum value is 0 . The search domain is $-5 \mathrm{~mm} \leq x, y \leq 5 \mathrm{~mm}$. The landscapes are: 
1) Sphere Function (Bowl-shaped):

$$
\begin{aligned}
& f(x, y)= \\
& \left\{\begin{aligned}
1, & \sqrt{x^{2}+y^{2}} \leq 0.5 \text { and }(x, y) \in \mathbb{V} \\
1-0.02\left(x^{2}+y^{2}\right), & \sqrt{x^{2}+y^{2}}>0.5 \text { and }(x, y) \in \mathbb{V} .
\end{aligned}\right.
\end{aligned}
$$
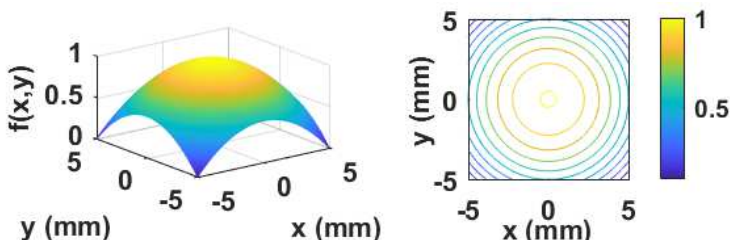

(a)

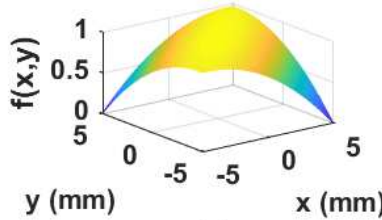

(c)

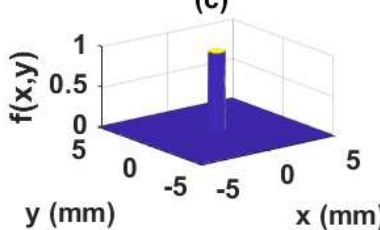

(e)

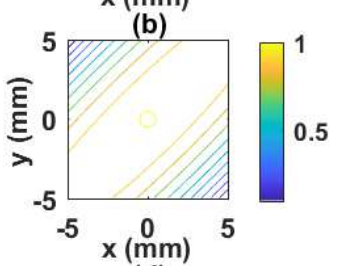

(d)

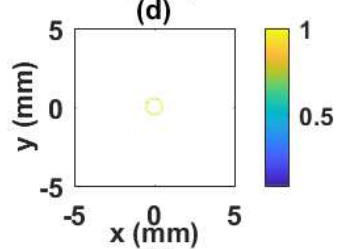

(f)

Fig. 8. Illustration of $f(x, y)$ for three representative objective functions: (a) Sphere function and (b) its contour plot; (c) Matyas function and (d) its contour plot; (e) Easom function and (f) its contour plot. For the objective $f(x, y)$, the maximum is normalized to 1 and the minimum value is 0 .

2) Matyas Function (Plate-shaped):

$$
\begin{aligned}
& f(x, y)= \\
& \left\{\begin{array}{r}
1, \\
1-0.01\left(x^{2}+y^{2}\right)+0.02 x y \\
\sqrt{x^{2}+y^{2}}>0.5 \text { and }(x, y) \in \mathbb{V} .
\end{array}\right.
\end{aligned}
$$

3) Easom function:

$$
\begin{aligned}
& f(x, y)= \\
& \left\{\begin{array}{l}
1, \quad \sqrt{x^{2}+y^{2}} \leq 0.5 \text { and }(x, y) \in \mathbb{V} \\
0.01+0.99 \cos (3 x) \cos (3 y) \\
\times \exp \left[-\left(9 x^{2}+9 y^{2}\right)\right], \\
\sqrt{x^{2}+y^{2}}>0.5 \text { and }(x, y) \in \mathbb{V} .
\end{array}\right.
\end{aligned}
$$

The term $\mathbb{V}$ denotes the discrete vascular network as illustrated in Fig. 3. As shown in Fig. 8(a)-(b), both the Sphere and Matyas functions represent the situation that the tumor center, denoted by a circle of radius $0.5 \mathrm{~mm}$ located at the origin, is associated with the region having the highest values of $f(x, y)$. This may correspond to the largest (normalized) variation of blood flow velocity due to tumor angiogenesis or the largest (normalized) magnetic change induced by nanoswimmers due to fibrin tropism in tumor tissues. Both the functions are convex and quadratic. They have no local minimum except the global one. On the other hand, the Easom function in Fig. 8(c) represents the situation that $f(x, y)$ remains more or

less unchanged across a large surveillance region. The tumor center yields an abrupt increase of $f(x, y)$. Intuitively, this may represent the worst-case direct targeting scenario due to the lack of an externally observable biophysical gradient.

In the absence of detailed information on the diameters of vessels, the viscosity of blood, and the applied blood pressure for tumor vessels and normal capillaries near the tumor, we simply imprint the objective functions in (7)-(9) on the vascular network $\mathbb{V}$. The blood inflow and outflow are assumed to be in the bottom left and top right, respectively, where prescribed pressures are set.

\section{GD-INSPIRED DTS}

The GD-inspired DTS starts with a generic guess $G_{1}$ located at $\vec{x}_{1}$ at time instant $t_{\mathrm{IA}, 1}^{(1)}$, which attempts to find a global maximum $f\left(\vec{x}^{*}\right)$.

\section{A. DTS for TDMI}

For TDM1 shown in Fig. 4(a), consider the sequence $\quad \vec{x}_{1}\left(t_{\mathrm{IT}, 1}^{(1)}\right), \vec{x}_{1}\left(t_{\mathrm{IT}, 1}^{(2)}\right), \vec{x}_{1}\left(t_{\mathrm{IT}, 1}^{(3)}\right), \cdots \quad$ In the classical $\mathrm{GD}, \quad \vec{x}_{1}\left(t_{\mathrm{IT}, 1}^{(m)}\right)=\vec{x}_{1}\left(t_{\mathrm{IT}, 1}^{(m-1)}\right)+$ $\gamma_{m-1} \nabla f\left(\vec{x}_{1}\left(t_{\mathrm{IT}, 1}^{(m-1)}\right)\right), m=2,3, \cdots$. In this way, we have $f\left(\vec{x}_{1}\left(t_{\mathrm{IT}, 1}^{(1)}\right)\right) \leq f\left(\vec{x}_{1}\left(t_{\mathrm{IT}, 1}^{(2)}\right)\right) \leq f\left(\vec{x}_{1}\left(t_{\mathrm{IT}, 1}^{(3)}\right)\right) \leq \cdots$, so hopefully the sequence $\vec{x}_{1}\left(t_{\mathrm{IT}, 1}^{(m)}\right)$ converges to the desired global maximum. However, in the GD-inspired DTS, the location updating is interrupted by multiple IT and UA processes as depicted in Fig. 4(a). Hence, the position update is modified as $\vec{x}_{1}\left(t_{\mathrm{IT}, 1}^{(m)}\right)=\vec{x}_{1}\left(t_{\mathrm{IA}, 1}^{(m)}\right)+\gamma_{m} \nabla f\left(\vec{x}_{1}\left(t_{\mathrm{IA}, 1}^{(m)}\right)\right)$.

The gradient $\nabla f\left(\vec{x}_{1}\left(t_{\mathrm{IA}, 1}^{(m)}\right)\right)$ is estimated through the $N$ IT processes as illustrated in Fig. 4(a). If the gradient does not change much over the duration of $t_{\mathrm{IT}, 1}^{(m)}$ to $t_{\mathrm{IT}, 1}^{(m+1)}$, it can be estimated as

$$
\nabla f\left(\vec{x}_{1}\left(t_{\mathrm{IA}, 1}^{(m)}\right)\right) \approx \max _{n=1,2, \cdots, N}\left\{\nabla f\left(\vec{x}_{1}\left(t_{\mathrm{IT}, n}^{(m)}\right)\right)\right\} .
$$

Otherwise, only the last gradient estimate is used such that

$$
\nabla f\left(\vec{x}_{1}\left(t_{\mathrm{IA}, 1}^{(m)}\right)\right) \approx \nabla f\left(\vec{x}_{1}\left(t_{\mathrm{IT}, N}^{(m)}\right)\right) .
$$

Suppose that $f(\vec{x})$ is convex and $\nabla f(\vec{x})$ is Lipschitz, the step size $\gamma_{m}$ can be chosen to guarantee convergence to a global optimum by using the Barzilai-Borwein method [46]:

$$
\begin{aligned}
\gamma_{m} \approx & \frac{\left(\vec{x}_{1}\left(t_{\mathrm{IA}, 1}^{(m)}\right)-\vec{x}_{1}\left(t_{\mathrm{IA}, 1}^{(m-1)}\right)\right)^{T}}{\left\|\nabla f\left(\vec{x}_{1}\left(t_{\mathrm{IA}, 1}^{(m)}\right)\right)-\nabla f\left(\vec{x}_{1}\left(t_{\mathrm{IA}, 1}^{(m-1)}\right)\right)\right\|^{2}} \\
& \times\left[\nabla f\left(\vec{x}_{1}\left(t_{\mathrm{IA}, 1}^{(m)}\right)\right)-\nabla f\left(\vec{x}_{1}\left(t_{\mathrm{IA}, 1}^{(m-1)}\right)\right)\right] .
\end{aligned}
$$

Note that the vessel network used in the simulation procedure is a discontinuous two-dimensional grid as shown in Fig. 3; therefore the position update follows the procedure described in Section III-B. As the vessels run only parallel to the two coordinate axes, at each junction the guess input can move in two possible directions, up and right, as the flow is from bottom left to top right. The Barzilai-Borwein condition 
in (12) is employed to determine the duration of the $m^{\text {th }}$ IA operation for $G_{1}$ :

$$
t_{\mathrm{IT}, 1}^{(m)}-t_{\mathrm{IA}, 1}^{(m)}=\frac{\gamma_{m} \cos \phi_{m}+\gamma_{m} \sin \phi_{m}}{v_{1}\left(t_{\mathrm{IA}, 1}^{(m)}\right)},
$$

where $\phi_{m}$ is the angle of the gradient estimated at the $m^{\text {th }}$ cycle.

\section{B. DTS for TDM2}

For TDM2 shown in Fig. 4(b), similarly, consider the sequence $\quad \vec{x}_{1}\left(t_{\mathrm{IA}, 2}^{(1)}\right), \vec{x}_{1}\left(t_{\mathrm{IA}, 2}^{(2)}\right), \vec{x}_{1}\left(t_{\mathrm{IA}, 2}^{(3)}\right), \cdots$. In the classical GD, $\vec{x}_{1}\left(t_{\mathrm{IA}, 2}^{(m)}\right)=\vec{x}_{1}\left(t_{\mathrm{IA}, 2}^{(m-1)}\right)+$ $\gamma_{m-1} \nabla f\left(\vec{x}_{1}\left(t_{\mathrm{IA}, 2}^{(m-1)}\right)\right), m=2,3, \cdots$, to ensure that the sequence $\vec{x}_{1}\left(t_{\mathrm{IA}, 2}^{(m)}\right)$ converges to the desired global maximum. However, in the GD-inspired DTS, the location updating is interrupted by multiple UA processes and one IT process as depicted in Fig. 4(b). Hence, the position update is expressed as $\vec{x}_{1}\left(t_{\mathrm{IA}, 2}^{(m)}\right)=\vec{x}_{1}\left(t_{\mathrm{IA}, 1}^{(m)}\right)+\gamma_{m} \nabla f\left(\vec{x}_{1}\left(t_{\mathrm{IA}, 1}^{(m)}\right)\right)$.

To ensure that such an arrangement does not favor IA processes that are closer to the earlier IT operation resulted from more accurate gradient estimation, the gradient change over the duration of $t_{\mathrm{IA}, 1}^{(m-1)}$ to $t_{\mathrm{IA}, 1}^{(m)}$ should be minimal, which is approximated by

$$
\nabla f\left(\vec{x}_{1}\left(t_{\mathrm{IA}, 1}^{(m)}\right)\right) \approx \nabla f\left(\vec{x}_{1}\left(t_{\mathrm{IT}}^{(m-1)}\right)\right),
$$

where $\nabla f\left(\vec{x}_{1}\left(t_{\mathrm{IT}}^{(m-1)}\right)\right)$ is the gradient estimated during the $(m-1)^{\text {th }}$ IT process.

Finally, due to the practical constraint of DTS, the initial deployment region of the guess input is confined within a small area, which is the injection site of nanoswimmers, instead of the entire solution space. To further ensure that the guess input is confined within the tissue region under screening, the replacement strategy is implemented: a guess that travels outside the allowed searching region is abandoned, which will degrade in the human body without further maneuvering and tracking. A new guess is then generated in the deployment area by injecting an aggregate of nanoswimmers.

\section{Performance Analysis}

We use several numerical examples to evaluate the tumor sensitization and targeting performance of the GD-inspired DTS, which is compared to the brute-force search.

For the DTS, both the two protocols in Fig. 4 are considered where two guess inputs are deployed for direct targeting. The durations of IA and IT are set to be $10 \mathrm{~s}$ and the number of observation intervals during each IT operation (see also Fig. 6 ) is 10 . The searching process is stopped if any guess reaches the cancer center denoted by a circle of radius $0.5 \mathrm{~mm}$ at the origin as shown in Fig. 8. It is assumed that the other guess can be guided to the tumor center upon successful detection if it has not overshot the tumor location.

For the brute-force search, each guess follows a random walk without learning from the environment, which synthesizes the contrast-enhanced medical imaging scenario where

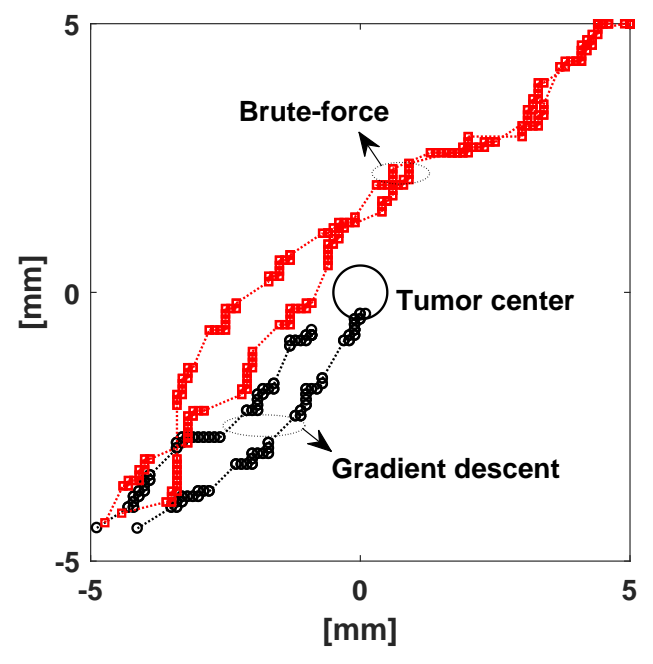

Fig. 9. Trajectories of guess inputs when TDM1 is applied: "o" - GD-inspired DTS, " $\square$ " - brute-force search.

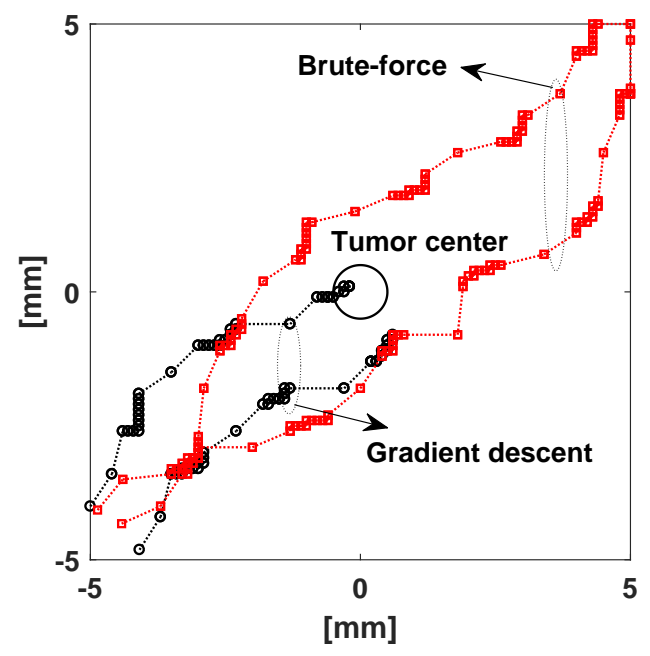

Fig. 10. Trajectories of guess inputs when TDM2 is applied: "o" - GDinspired DTS, " $\square$ " - brute-force search.

contrast agent nanoparticles attempt to target a tumor by enumerating all possible pathways in the vascular network. For consistency with the GD-inspired DTS, the trajectories of two guess inputs are displayed. Furthermore, the random drift and IT durations follow the two protocols in Fig. 4, though the movement of these two guesses are completely independent of each other. The searching process is stopped if any guess reaches the cancer center as in the case of DTS. However, the other guess will continue its random drift in the absence of an external guidance.

The three objective functions presented in Section III-C are applied to synthesize different levels of tumor sensitization difficulty. The initial deployment region is $-5 \mathrm{~mm} \leq x, y \leq$ $-4 \mathrm{~mm}$. The speed of nanoswimmers is $100 \mu \mathrm{m} / \mathrm{s}$. The maximum search time allowed is $200 \mathrm{~s}$ and the total number of simulation runs is 1000 . Two performance metrics are 
considered, the probability of cancer detection $P_{D}$ and the percentage of contrast agent nanoparticles delivered to the tumor site $\eta$.

Fig. 9 shows the typical trajectories of guess inputs for the landscape of Sphere function when the TDM1 protocol is considered. The symbols of " $\circ$ " and " $\square$ " denote the actual guess footprints for the GD-inspired DTS and brute-force search, respectively, and regions with clustered footprints correspond to the IA mode. As can be seen from the figure, in the case of DTS the movement of both guesses is coordinated by an external field towards the maximum-gradient direction estimated in the IA mode. On the other hand, the movement of two guesses is irregular and uncorrelated for the bruteforce search. The DTS successfully detects the tumor center, whereas the brute-force search technique fails to find the center of tissue malignancy even with multiple guesses. Fig. 10 presents the guess trajectories when the TDM2 protocol is applied. The time interval between two consecutive IAs for TDM2 is twice of the value for TDM1 because in the former case, each guess takes turn to operate in the IA mode, followed by a common IT mode. Hence, the gradient estimated during the IT operation may be different from the actual gradient for the later IA process, leading to more departing trajectories of the guesses as depicted in Fig. 10. It is expected that this phenomenon would result in deteriorating tumor sensitization and targeting performance. Similar observations were made for the Matyas and Easom landscapes.

Fig. 11 presents the histograms of search time for the three objective functions when the TDM1 protocol is employed. The search time of $200 \mathrm{~s}$ (maximum value) indicates the situation that none of the two guesses senses the tumor. It can be seen that the GD-inspired DTS yields a detection ratio of $P_{D}=89.6 \%$ for the Sphere function (Fig. 11(a)), which is much higher than that for the brute-force search $\left(P_{D}=58.1 \%\right.$, Fig. 11(b)). Furthermore, the DTS has better performance in the Sphere landscape than the Matyas $\left(P_{D}=73.0 \%\right.$, Fig. 11(c)) and Easom $\left(P_{D}=59.8 \%\right.$, Fig. 11(e)) functions. This observation demonstrates the advantage of the proposed biosensing-by-learning strategy over brute-force search and the potential performance deterioration due to a more complex landscape (i.e., plate-shaped Matyas function and gradientless Easom function versus bowl-shaped Sphere function). In terms of the targeting efficiency, the DTS achieves a much higher value of $\eta=78.0 \%$ compared to that for the Matyas $(\eta=61.6 \%)$ and Easom $(\eta=49.0 \%)$ functions as well as the brute-force search $(\eta \approx 32 \%)$.

Fig. 12 presents the histograms of search time for TDM2. Similar observations to the trend in Fig. 11 can be made. Furthermore, comparing Fig. 12 to Fig. 11 shows that, TDM1 yields higher probabilities of tumor sensitization and larger percentages of drug molecules delivered to the tumor than TDM2.

\section{CONCLUSION}

We have proposed a novel iterative-optimization-inspired DTS in externally manipulable smart nanosystems, which exploits tumor-triggered in vivo biophysical gradients for

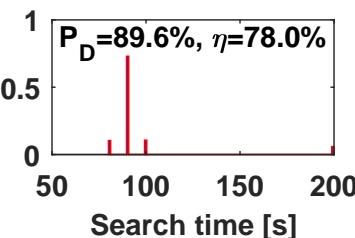

(a)

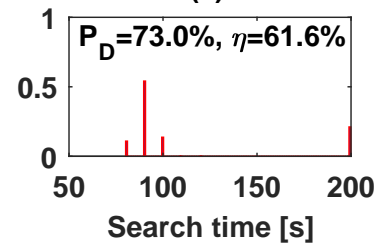

(c)

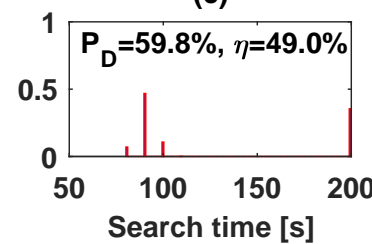

(e)

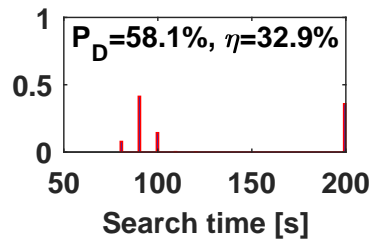

(b)

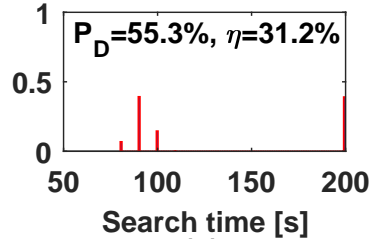

(d)

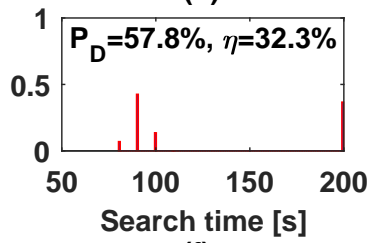

(f)
Fig. 11. Histograms of search time when TDM1 is applied: (a) GD-inspired DTS and (b) brute-force search in a Sphere landscape; (c) GD-inspired DTS and (d) brute-force search in a Matyas landscape; (c) GD-inspired DTS and (d) brute-force search in an Easom landscape. Also shown are the respective detection ratios $P_{D}$ and targeting efficiencies $\eta$.

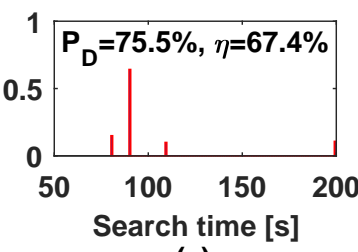

(a)

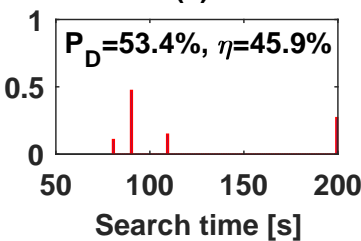

(c)

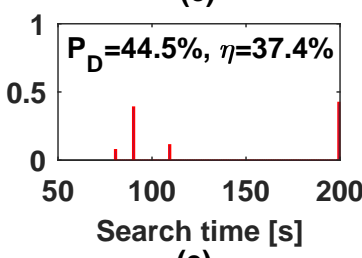

(e)

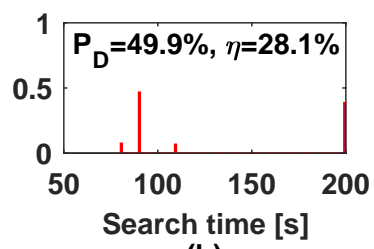

(b)

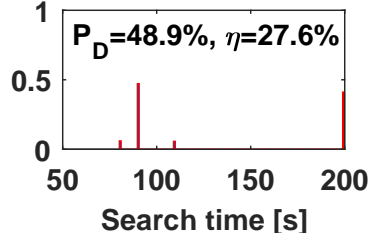

(d)

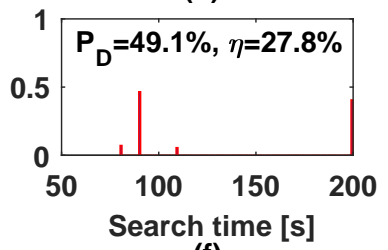

(f)
Fig. 12. Histograms of search time when TDM2 is applied: (a) GD-inspired DTS and (b) brute-force search in a Sphere landscape; (c) GD-inspired DTS and (d) brute-force search in a Matyas landscape; (c) GD-inspired DTS and (d) brute-force search in an Easom landscape. Also shown are the respective detection ratios $P_{D}$ and targeting efficiencies $\eta$. 
"guided" direct targeting. We have demonstrated through computational experiments that the proposed DTS can significantly improve the probability of tumor sensitization and the accumulation of drug nanoparticles in the tumor site by using the shortest possible physiological routes and with minimal systemic exposure. We believe that this work motivates a new paradigm directed toward smart biosensing facilitated by externally controllable nanoswimmers.

Future work may include extension of the framework to DTS inspired by multi-solution or multi-objective optimizations when there are multiple tumors or different phenomenaof-interest in the tissue region under surveillance. Moreover, it is important to examine further the impact of nanoswimmer nonidealities, such as finite lifespan, imprecise steering, and inaccurate tracking. Finally, the proposed DTS and the objective functions used should be validated by real experiments to justify further the clinical relevance of the proposed strategy.

\section{REFERENCES}

[1] S. Thoidingjam and A. B. Tiku, "New developments in breast cancer therapy: role of iron oxide nanoparticles," Adv. Nat. Sci.: Nanosci. Nanotechnol., vol. 8, pp. 023002, 2017.

[2] A. Modiri, S. Goudreau, A. Rahimi, and K. Kiasaleh, "Review of breast screening: Toward clinical realization of microwave imaging," Med. Phys., vol. 44, no. 12, pp. e446-e458, 2017.

[3] F. Yang, L. Sun, Z. Hu, H. Wang, D. Pan, R. Wu, X. Zhang, Y. Chen, and Q. Zhang, "A large-scale clinical trial of radar-based microwave breast imaging for Asian women: Phase I," in Proc. IEEE IEEE AP$S$ Symposium on Antennas and Propagation and USNC-URSI Radio Science Meeting 2017, San Diego, USA, July 2017.

[4] H. Song, S. Sasada, T. Kadoya, M. Okada, K. Arihiro, X. Xiao, and T. Kikkawa, "Detectability of breast tumor by a hand-held impulseradar detector: Performance evaluation and pilot clinical study," Sci. Rep., vol. 7, no. 1, pp. 16353, 2017.

[5] E. Porter, M. Coates, and M. Popović, "An early clinical study of timedomain microwave radar for breast health monitoring," IEEE Trans. Biomed. Eng., vol. 63, no. 3, pp. 530-539, 2016.

[6] A. W. Preece, I. Craddock, M. Shere, L. Jones, and H. L. Winton, "MARIA M4: Clinical evaluation of a prototype ultrawideband radar scanner for breast cancer detection," J. Med. Imag., vol. 3, no. 3, pp. 033502, 2016

[7] P. M. Meaney, P. A. Kaufman, L. S. Muffly, M. Click, S. P. Poplack, W. A. Wells, G. N. Schwartz, R. M. di Florio-Alexander, T. D. Tosteson, Z. Li, S. D. Geimer, M. W. Fanning, T. Zhou, N. R. Epstein, and K. D. Paulsen, "Microwave imaging for neoadjuvant chemotherapy monitoring: Initial clinical experience," Breast Cancer Res., vol. 15, no. 2, pp. 1-16, 2013

[8] D. O'Loughlin, M. J. O'Halloran, B. M. Moloney, M. Glavin, E. Jones, and M. A. Elahi, "Microwave breast imaging: Clinical advances and remaining challenges," IEEE Trans. Biomed. Eng., 2018, Early Access, DOI: 10.1109/TBME.2018.2809541.

[9] M. Lazebnik, D. Popovic, L. McCartney, C. B.Watkins, M. J. Lindstrom, J. Harter, S. Sewall, T. Ogilvie, A. Magliocco, T. M. Breslin, W. Temple, D. Mew, J. H. Booske, M. Okoniewski, and S. C. Hagness, "A large-scale study of the ultrawideband microwave dielectric properties of normal, benign and malignant breast tissues obtained from cancer surgeries," Phys. Med. Biol., vol. 52, pp. 6093-6115, 2007.

[10] A. P. O'Rourke, M. Lazebnik, J. M. Bertram, and et al., "Dielectric properties of human normal, malignant and cirrhotic liver tissue: in vivo and ex vivo measurements from 0.5 to $20 \mathrm{GHz}$ using a precision openended coaxial probe," Phys. Med. Biol., vol. 52, pp. 4707-4719, 2007.

[11] S. Semenov, "Microwave tomography: Review of the progress towards clinical applications," Phil. Trans. R. Soc. A, vol. 367, pp. 3021-3042, 2009.

[12] O. M. Bucci, G. Bellizzi, A. Borgia, S. Costanzo, L. Crocco, G. Di Massa, and R. Scapaticci, "Experimental framework for magnetic nanoparticles enhanced breast cancer microwave imaging," IEEE Access, vol. 5, pp. 16332-16340, 2017.
[13] O. M. Bucci, L. Crocco, and R. Scapaticci, "On the optimal measurement configuration for magnetic nanoparticles-enhanced breast cancer microwave imaging," IEEE Trans. Biomed. Eng., vol. 62, no. 2, pp. 407-414, 2015

[14] Y. H. Bae and K. Park, "Targeted drug delivery to tumors: Myths, reality and possibility," J. Con. Rel., vol. 153, pp. 198-205, 2011.

[15] O. Felfoul, M. Mohammadi, S. Taherkhani, and et al., "Magnetoaerotactic bacteria deliver drug-containing nanoliposomes to tumour hypoxic regions," Nature Nanotechnology, vol. 11, pp. 941-947, 2016.

[16] U. K. Cheang and M. J. Kim, "Self-assembly of robotic micro- and nanoswimmers using magnetic nanoparticles," J. Nanopart. Res., vol. 17, no. 145, 2015.

[17] U. K. Cheang, F. Meshkati, H. Kim, K. Lee, H. C. Fu, and M. J. Kim "Versatile microrobotics using simple modular subunits," Sci. Rep., vol. 6, no. 30472, 2016.

[18] U. K. Cheang, H. Kim, D. Milutinović, J. Choi, and M. J. Kim, "Feedback control of robotic achiral microswimmers," J. Bionic. Eng. vol. 14, no. 2, pp. 245-259, 2017.

[19] L. Mertz, "Tiny conveyance: Micro- and nanorobots prepare to advance medicine," IEEE Pulse, vol. 9, no. 1, pp. 19-23, 2018.

[20] K. Seidi, H. A. Neubauer, R. Moriggl, and R. Jahanban-Esfahlan, "Tumor target amplification: Implications for nano drug delivery systems," J. Con. Rel., vol. 275, pp. 142-161, 2018.

[21] M. Overchuk and G. Zheng, "Overcoming obstacles in the tumor microenvironment: Recent advancements in nanoparticle delivery for cancer theranostics," Biomaterials, vol. 156, pp. 217-237, 2018.

[22] H. Kim, J. Lee, C. Oh, and J.-H. Park, "Cooperative tumour cell membrane targeted phototherapy," Nat. Commun., vol. 8, no. 15880, pp. 1-10, 2017.

[23] B. Zhang, H. Wang, S. Shen, X. She, W. Shi, J. Chen, Q. Zhang, Y. Hu, Z. Pang, and X. Jiang, "Fibrin-targeting peptide creka-conjugated multi-walled carbon nanotubes for self-amplified photothermal therapy of tumor," Biomaterials, vol. 79, pp. 46-55, 2016.

[24] E. J. Kwon, J. H. Lo, and S. N. Bhatia, "Smart nanosystems: Bioinspired technologies that interact with the host environment," Proc. Natl. Acad. Sci. U. S. A., vol. 112, no. 47, pp. 14460-14466, 2015.

[25] G. von Maltzahn, J.-H. Park, K. Y. Lin, N. Singh, C. Schwöppe, R. Mesters, W. E. Berdel, E. Ruoslahti, M. J. Sailor, and S. N. Bhatia, "Nanoparticles that communicate in vivo to amplify tumour targeting," Nat. Mater., vol. 10, pp. 545-552, 2011.

[26] J.-H. Park, G. von Maltzahn, L. L. Ong, A Centrone, T. A. Hatton, E. Ruoslahti, S. N. Bhatia, and M. J. Sailor, "Cooperative nanoparticles for tumor detection and photothermally triggered drug delivery," $A d v$. Mater., vol. 22, pp. 880-885, 2010.

[27] L. Agemy, K. N. Sugahara, V. R. Kotamraju, K. Gujraty, O. M. Girard, Y. Kono, R. F. Mattrey, J.-H. Park, M. J. Sailor, A. I. Jimenez, C. Cativiela, D. Zanuy, F. J. Sayago, C. Aleman, R. Nussinov, and E. Ruoslahti, "Nanoparticle-induced vascular blockade in human prostate cancer," Blood, vol. 116, pp. 2847-2856, 2010.

[28] J.-H. Park, G. von Maltzahnc, M. J. Xu, V. Fogald, V. R. Kotamraju, E. Ruoslahti, S. N. Bhatia, and M. J. Sailor, "Cooperative nanomaterial system to sensitize, target, and treat tumors," Proc. Natl. Acad. Sci. U. S. A., vol. 107, no. 3, pp. 981-986, 2010.

[29] D. Simberg, T. Duza, J.-H. Park, M. Essler, J. Pilch, L. Zhang, A. M. Derfus, M. Yang, R. M. Hoffman, S. Bhatia, M. J. Sailor, and E. Ruoslahti, "Biomimetic amplification of nanoparticle homing to tumors," Proc. Natl. Acad. Sci. U. S. A., vol. 104, no. 3, pp. 932-936, 2007.

[30] Y. Chen, S. Shi, X. Yao, and T. Nakano, "Touchable computing: Computing-inspired bio-detection," IEEE Trans. Nanobiosci., vol. 16, no. 8, pp. 810-821, 2017.

[31] Y. Chen, T. Nakano, P. Kosmas, C. Yuen, A. V. Vasilakos, and M. Asvial, "Green touchable nanorobotic sensor networks," IEEE Commun. Mag., pp. 136-142, Nov. 2016.

[32] Y. Chen, P. Kosmas, and S. Martel, "A feasibility study for microwave breast cancer detection using contrast-agent-loaded bacterial microbots," Int. J. Antennas Propag., vol. 2013, Article ID 309703, 11 pages, http://dx.doi.org/10.1155/2013/309703.

[33] Y. Gazit, D. A. Berk, M. Leunig, L. T. Baxter, and R. K. Jain, "Scaleinvariant behavior and vascular network formation in normal and tumor tissue," Phys. Rev. Lett., vol. 75, no. 12, pp. 2428-2431, 1995.

[34] J. W. Baish, Y. Gazit, D. A. Berk, M. Nozue, L. T. Baxter, and R. K. Jain, "Role of tumor vascular architecture in nutrient and drug delivery: An invasion percolation-based network model," Microvasc. Res., vol. 51, pp. 327-346, 1996.

[35] J. W. Baish and R. K. Jain, "Fractals and cancer," Cancer Res., vol. 60, pp. 3683-3688, 2000. 
[36] D. Fukumura and R. K. Jain, "Tumor microvasculature and microenvironment: targets for anti-angiogenesis and normalization," Microvasc. Res., vol. 74, pp. 72-84, 2007.

[37] Y. Wang, K. Iguchi, H. Ito, K. Ookawa, N. Kobayashi, R. Nakamura, Y. Goto, M. Sakai, S. Ishikawa, and M. Onizuka, "Blood flow velocity is reduced in a tumor micro-dissemination in the visceral pleura in anesthetized open-chest rat lung," In Vivo, vol. 23, pp. 291-296, 2009.

[38] G. Komar, S. Kauhanen, K. Liukko, M. Seppanen, S. Kajander, J. Ovaska, P. Nuutila, and H. Minn, "Decreased blood flow with increased metabolic activity: A novel sign of pancreatic tumor aggressiveness," Clin. Cancer Res., vol. 15, no. 17, pp. 5511-5517, 2009.

[39] T. E. Dudar and R. K. Jain, "Differential response of normal and tumor microcirculation to hyperthermia," Cancer Res., vol. 44, pp. 605-612, 1984.

[40] C. W. Song, "Effect of local hyperthermia on blood flow and microenvironment: A review," Cancer Res. (Suppl.), vol. 44, pp. 4721s-4730s, 1984.

[41] D.-S. Lee, H. Rieger, and K. Bartha, "Flow correlated percolation during vascular remodeling in growing tumors," Phys. Rev. Lett., vol. 96, no. 058104, 2006.

[42] S. R. McDougall, A. R. A. Anderson, and M. A. J. Chaplain, "Mathematical modelling of flow through vascular networks: Implications for tumor-induced angiogenesis and chemotherapy strategies," Bull. Math. Biol., vol. 64, pp. 673-702, 2002.

[43] R. M. Rangayyan, N. M. El-Faramawy, J. E. Leo Desautels, and O. A. Alim, "Measures of acutance and shape for classification of breast tumors," IEEE Trans. Med. Imag., vol. 16, no. 6, pp. 799-810, 1997.

[44] R. Saunders, E. Samei, J. Baker, and D. Delong, "Simulation of mammographic lesions," Academic Radiology, vol. 13, no. 7, pp. 860870,2006

[45] Y. Chen, P. Kosmas, P. S. Anwar, and L. Huang, "A touchcommunication framework for drug delivery based on a transient microbot system," IEEE Trans. Nanobiosci., vol. 14, no. 4, pp. 397-408, June 2015 .

[46] J. Barzilai and J. M. Borwein, "Two-point step size gradient methods," IMA Journal of Numerical Analysis, vol. 8, no. 1, pp. 141-148, 1988. 